Rev. Elev. Méd. vét. Pays trop., 1973, 26 (4) : 21 a - 48 a

\title{
Bases anatomiques et fonctionnelles de la reproduction chez le zébu (Bos indicus)
}

\author{
par P. CUQ (*)
}

\begin{abstract}
RESUME
Le rapport présenté par l'auteur a été élaboré à partir d'observations publiées par les zootechniciens qui s'occupent de la reproduction du zébu dans les aires d'extension de cette espèce et de travaux effectués au Laboratoire d'Anatomic, Histologie et Embryologie de l'Institut des Sciences et Médecine Vétérinaires de l'Université de Dakar.

Dans un chapitre consacré à l'anatomie, les particularités du tractus génital du mâle et de la femelle sont notées.

L'aspect fonctionnel du problème fait l'objet du second chapitre dans lequel sont rapportées d'abord les données zootechniques ayant trait à la durée du cycle oestral, à l'cestrus, aux âges auxquels se produisent la puberté, la première saillie féconde et le premier vêlage, à la durée de la gestation, à l'intervalle qui sépare deux vêlages consécutifs, au rythme annuel des fécondations et aux facteurs saisonniers capables d'influencer la reproduction.

L'étude histo-physiologique a permis de mettre en évidence des phases d'anœstrus chez les femelles vides et trois générations successives de corps gestatifs sur les femelles pleines. Chez les mâles, l'auteur signale des périodes caractérisées par des images de repos des glandes annexes du tractus génital.
\end{abstract}

Un nombre relativement important de travaux ont été consacrés à la reproduction du zébu. Ils se rapportent à des populations animales localisées dans toutes les aires d'extension de cette espèce : Afrique occidentale, Afrique orientale, Afrique du Sud, Australie, Amérique, Indes... La plupart sont l'œuvre de zootechniciens dont les buts et les méthodes d'investigation sont particuliers à leur spécialité, de sorte que, si la littérature est riche en observations se rapportant aux techniques d'élevage, elle est, par contre, pratiquement muette sur les particularités anatomiques, histologiques et physiologiques qui caractérisent le tractus génital de cette espèce.

Le présent rapport a été réalisé à partir des données de la littérature mais il tient compte aussi des résultats de recherches effectuées depuis 4 ans au Laboratoire d'Anatomie, Histologie et Embryologie de l'Ecole Inter-Etats des Sciences et Médecine Vétérinaires de l'Université de Dakar. Il n'a pas d'autres prétentions que celle de faire le point des connaissances acquises dans les domaines de la morphologie et du fonctionnement du tractus génital du mâle et de la femelle zébu.

\section{CARACTERISTIQUES MORPHOLOGIQUES DE L'APPAREIL GENITAL DU ZEBU}

Respectant les subdivisions classiques de l'anatomie descriptive, nous signalerons successivement les particularités des portions glandulaires, tubulaires et copulatrices des appareils génitaux du mâle et de la femelle.

(*) Professeur à l'Ecole Inter-Etats des Sciences et Médecine Vétérinaires, Service d'Anatomie, Histologie et Embryologie, Dakar, Sénégal. 


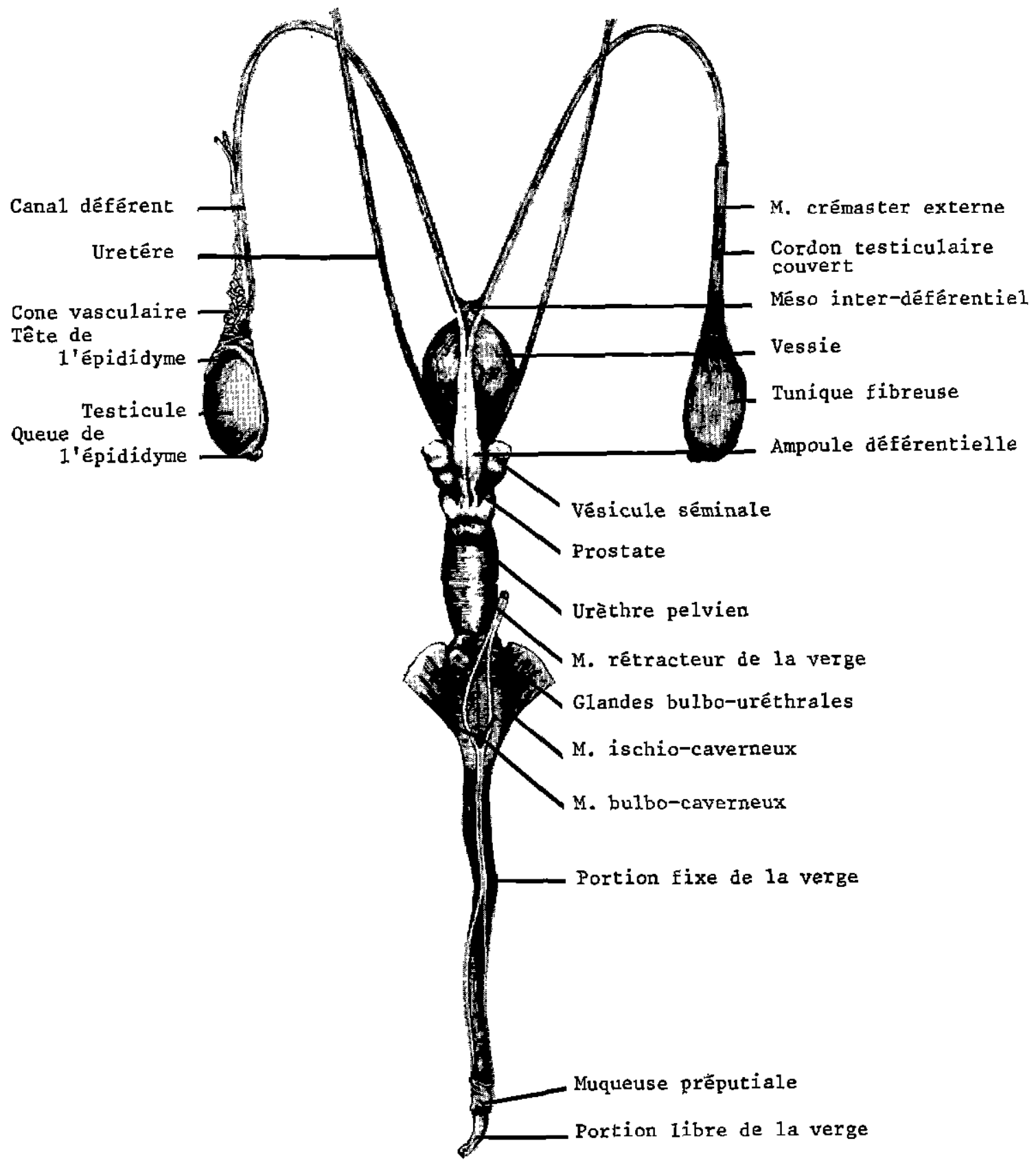

TRACTUS GENITAL DU TAUREAU ZEBU

(Vue dorsale après isolement) 


\section{I.A.) Caractéristiques morphologiques de l'appareil génital mâle}

\section{I.A.1.) PORTION GLANDULAIRE ET FORMATIONS ANNEXES}

Le zébu est exorchide (ou phanerorchide). A la fin de leur migration, les testicules sont en position sous-inguinale. Des enveloppes dites bourses testiculaires les protègent.

\section{I.A.1.a) Bourses testiculaires}

Des enveloppes qui entourent le testicule et la portion inguinale des voies spermatiques, certaines sont des dépendances du revêtement cutané; elles constituent les bourses tégumentaires (Scrotum) qui comprennent un revêtement cutané scrotal (Cuti scroti) et un peaucier à fibres lisses, le dartos (Dartos). Les autres se développent au moment même de la migration testiculaire. Elles forment les bourses profondes ou intra-inguinales (Tunicae funiculi spermatici et testis) qui comprennent le fascia lamelleux (Fascia spermatica externa), le muscle crémaster externe (M. Cremaster), la tunique fibreuse (Fascia spermatica interna) et la tunique séreuse (Tunica vaginalis).

Le SCROTUM CUTANE correspond au revêtement tégumentaire. Il forme un sac ovoïde, allongé dorso-ventralement et légèrement aplati cranio-caudalement, dans lequel les testicules, l'épididyme et le canal déférent occupent une position verticale. Ce sac tégumentaire est appendu à la région inguinale par un long col qui facilite la palpation et l'accès chirurgical aux organes qu'il contient.

La peau qui le constitue est souple et recouverte de poils fins et courts. Elle est marquée par un sillon cranio-caudal qui subdivise le sac cutané en deux lobes correspondant à chacune des glandes. Juste en avant du sac scrotal, on note sur la paroi abdominale la présence de quatre trayons rudimentaires.

Très riche en glandes sudoripares de type apocrine, la peau des bourses testiculaires joue un rôle important dans la régulation thermique de la glande génitale.

Le DARTOS (Tunica Dartos) double le revêtement cutané. Pour chaque testicule et son cordon, il forme un long sac qui remonte jusqu'à l'anneau inguinal superficiel, s'étend en avant vers le fourreau et, en arrière, se prolonge en direction du périné. Dans le plan médian, les deux sacs dartoïques s'affrontent et forment une cloison : le septum scrotal (Septum scroti).

La tunique dartö̈que est constituée de fibres musculaires lisses et de tissu conjonctif dense. Sa face externe adhère intimement à la peau. Homologue d'un muscle peaucier, elle entraîne le revêtement cutané et mobilise secondairement le testicule lorsqu'elle se contracte. Sa face interne par contre est très mobile par rapport aux enveloppes profondes. Cette particularité jointe à l'absence de ligament fibro-dartoïque ou scrotal (Ligamentum scroti) permet une mobilisation facile de la glande et de ses enveloppes profondes par rapport aux bourses cutanées. Elle rend possible chez le zébu la manceuvre du bistournage.

Le FASCIA LAMELLEUX (Fascia spermatica externa) double la face profonde du dartos et le sépare des bourses intra-inguinales. Il est formé de lamelles conjonctives superposées qui glissent facilement les unes sur les autres.

Le MUSCLE CREMASTER ( $M$. cremaster) est une dépendance du muscle oblique interne de l'abdomen. Relativement peu développé, il recouvre la face externe de la tunique fibreuse. Son insertion proximale s'effectue sur l'anneau inguinal profond. Distalement, il se termine en se fixant à la partie supérieure de la portion élargie du sac fibreux.

La TUNIQUE FIBREUSE (Fascia spermatica interna) forme un sac très allongé qui s'étend de l'anneau inguinal profond aux bourses cutanées. Son fond renflé loge le testicule, l'épididyme et la portion testiculaire du canal déférent. Sa partie supérieure, plus étroite, forme un goulot ou collet très long qui se fixe sur l'anneau inguinal profond et se confond à ce niveau avec le Fascia transversalis; elle renferme la portion inguinale du canal déférent. 

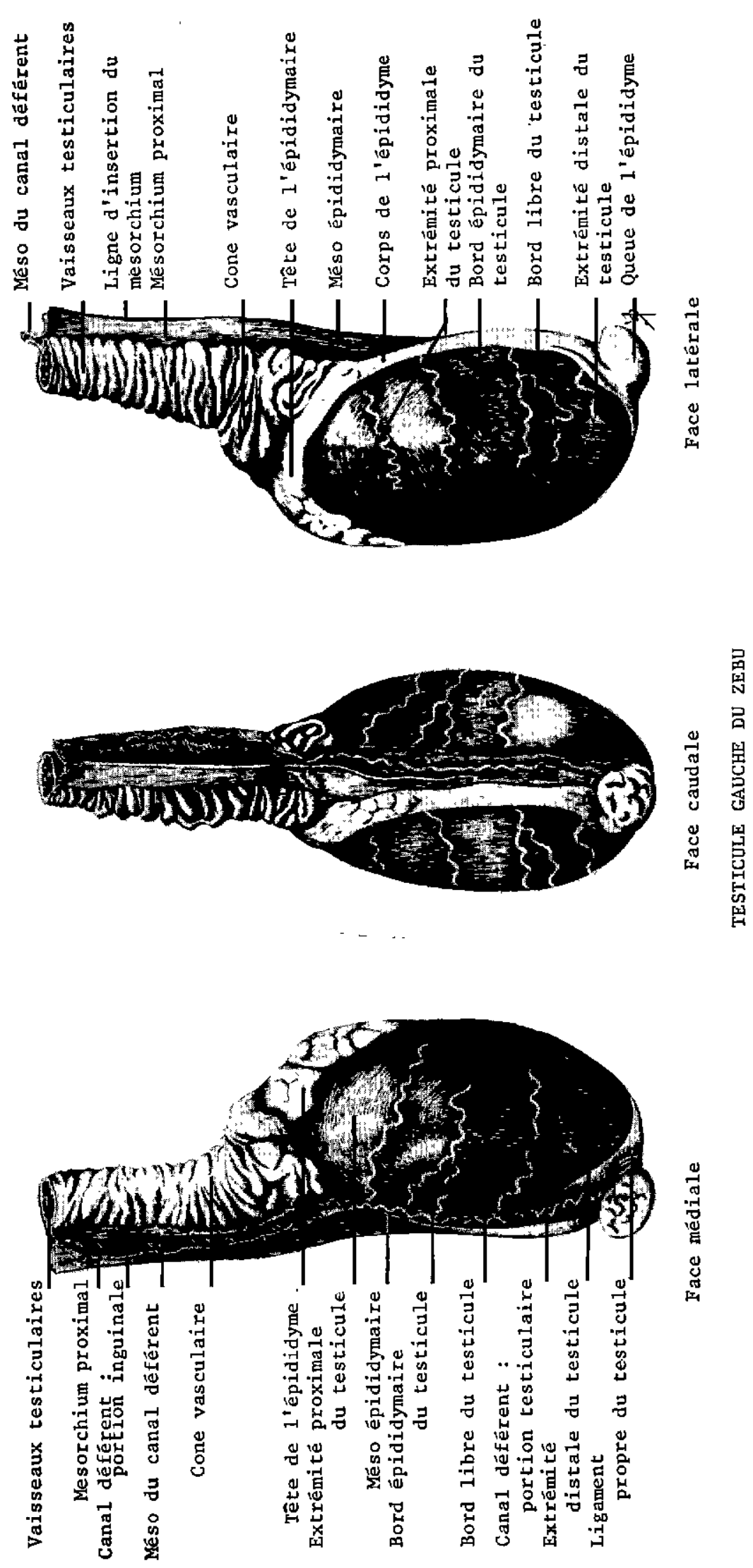
La TUNIQUE SEREUSE OU VAGINALE (Tunica vaginalis) est une dépendance du péritoine dont les divers éléments ont accompagné le testicule dans sa migration.

Le feuillet pariétal (Lamina parietalis) adhère très intimement à la face interne de la tunique fibreuse. Au niveau de l'anneau inguinal profond, il forme l'anneau vaginal (Anulus vaginalis) aussi réduit en dimension que l'anneau inguinal qui le contient.

Le feuillet viscéral (Lamina visceralis) tapisse la glande testiculaire et ses annexes. Il est uni au feuillet pariétal par le Mesorchium dont l'insertion s'effectue le long du bord postérieur du sac fibreux.

La cavité séreuse ainsi délimitée, véritable dépendance de la cavité péritonéale, constitue le canal vaginal (Canalis vaginalis) dans la région qui correspond au goulot de la fibreuse, et la cavité vaginale (Cavum vaginale) dans la région du fond.

\section{I.A.1.b) Les Testicules}

Les TESTICULES (Testes) du zébu sont ovoïdes, allongés et légèrement aplatis d'un côté à l'autre. Dans les bourses, ils sont disposés de telle façon que leur grand axe est vertical.

L'extrémité supérieure (Extremitas capitata) reçoit médialement l'insertion đu cône vasculaire. Elle est en continuité avec la tête de l'épididyme qui occupe une position latérale.

L'extrémité inférieure (Extremitas caudata) est en rapport avec la queue de l'épididyme à laquelle l'unit le court ligament propre du testicule (Lig. testis proprium).

Les FACES LATERALE ET MEDIALE sont convexes et lisses. On y observe des vaisseaux superficiels à trajet sinueux.

Le bord libre (Margo liber) est cranial. Le bord épididymaire (Margo epididymalis) est caudal. Le corps de l'épididyme et la portion testiculaire du canal déférent y adhèrent intimement.

L'ALBUGINEE (Tunica albuginea) est mince et les cloisons (Septula testis) que délègue sa face interne vers le corps d'Higmore (Mediastinum testis), discrètes.

Le PARENCHYME TESTICULAIRE (Parenchyma testis), subdivisé par les septa en lobules (Lobuli testis), est de coloration grisâtre. Il est formé de tubes séminifères (Tubuli seminiferi) et de cellules de la glande interstitielle réparties dans une trame conjonctivo-vasculaire délicate.

Les tubes séminifères sont très fortement circonvolutionnés (Tubuli seminiferi contorti). Au sommet de chaque lobule, ils sont collectés par des tubes d'abord courts et rectilignes (Tubuli seminiferi recti) puis anastomosés irrégulièrement entre eux dans le Mediastinum testis où ils constituent le Rete testis.

Les canaux efférents du testicule (Ductuli efferentes testis) procèdent du Rete testis et pénètrent dans la tête de l'épididyme.

Les cellules interstitielles ou cellules de Leydig, polyédriques, sont habituellement groupées en îlots dans les travées conjonctivo-vasculaires qui séparent les tubes séminifères.

\section{I.A.2.) PORTION TUBULAIRE DE L'APPAREIL GENITAL MALE}

\section{I.A.2.a) L'épididyme}

L'épididyme (Epididymis) est un organe allongé qui coiffe les extrémités et longe le bord caudal du testicule.

La TETE DE L'EPIDIDYME (Caput epididymis) adhère à l'extrémité supérieure de la gonade avec laquelle elle est en continuité de substance. Elle occupe une position latérale par rapport au cône vasculaire et se réfléchit très en avant sur le bord libre de la glande. 


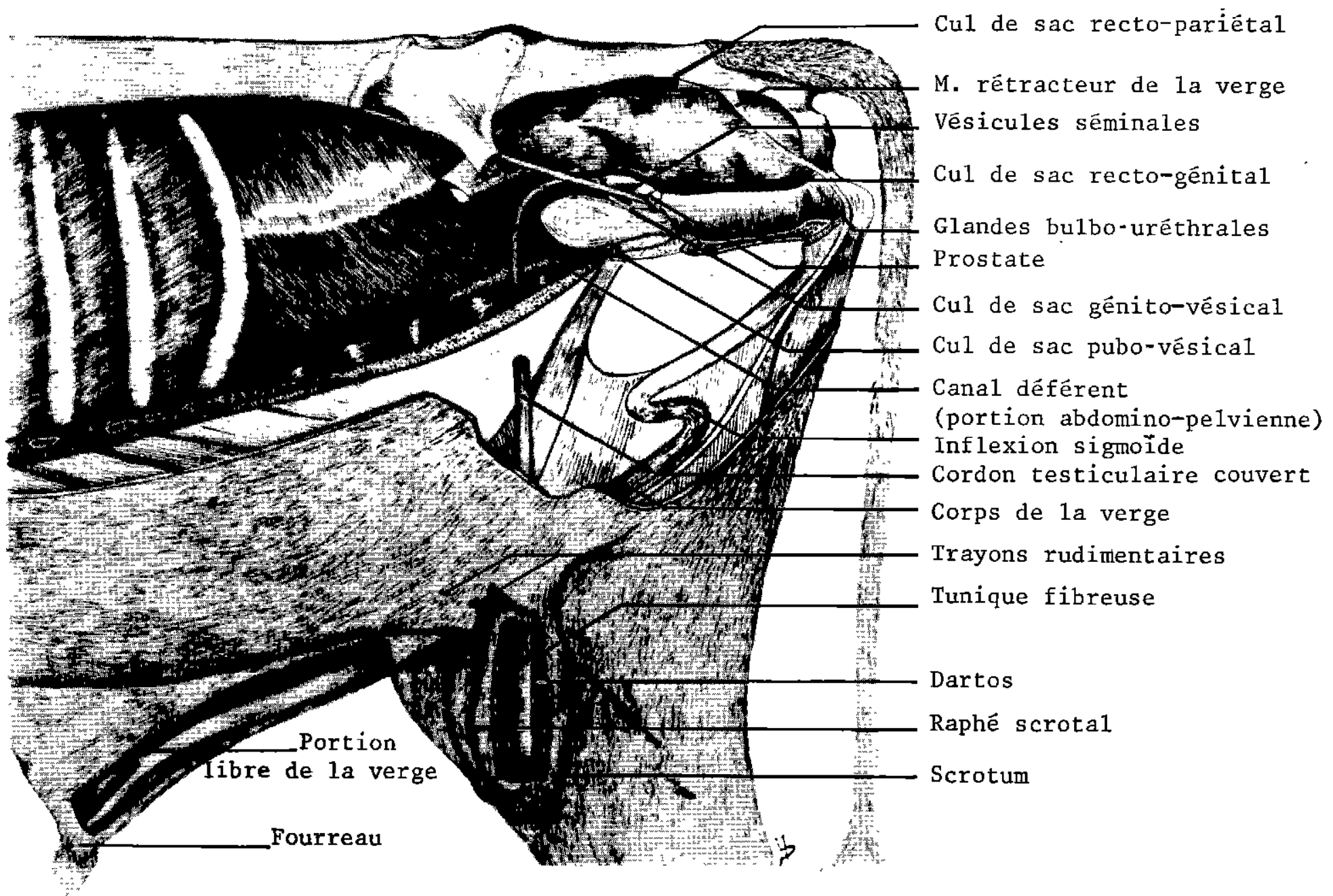

LE TRACTUS GENITAL DU TAUREAU ZEBU

(Vue latérale gauche des organes en place) 
Le CORPS DE L'EPIDIDYME (Corpus epididymis) longe, légèrement en dehors, le bord caudal fixe ou épididymaire du testicule. Il reçoit en arrière l'insertion du méso-épididymaire (Mesoepididymis) et adhère à l'albuginée testiculaire de telle façon que la bourse testiculaire (Bursa testicularis) et le mésorchium distal (Mesorchium distale) qui la limite médialement sont virtuels chez le zébu.

La QUEUE DE L'EPIDIDYME (Cauda epididymis) contourne de dehors en dedans l'extrémité caudale du testicule et se prolonge par le canal déférent. Elle est unie à l'albuginée testiculaire par un solide ligament propre du testicule (Lig. testis proprium), mais ne contracte aucune adhérence avec la fibreuse, par suite de la disparition du ligament de la queue de l'épididyme (Lig. caudae epididymis).

L'ALBUGINEE EPIDIDYMAIRE est en continuité de substance avec l'albuginée testiculaire. Relativement épaisse au niveau de la tête, elle lance des cloisons incomplètes qui tendent à subdiviser cette partie de l'épididyme en lobules (Lobuli epididymis). Au niveau du corps et de la queue, l'albuginée s'amincit progressivement. Elle se prolonge distalement par le mince conjonctif sous-séreux du canal déférent.

Le CANAL EPIDIDYMAIRE (Ductus epididymis) très long et sinueux prolonge les canaux efférents du testicule. Sa paroi comprend un épithélium qui repose sur une vitrée doublée d'un chorion et d'une couche musculaire. L'épithélium est formé de cellules basales petites et disséminées et d'une assise simple de hautes cellules cylindriques ciliées et glandulaires.

\section{I.A.2.b) Le canal déférent et le cordon testiculaire}

Le canal déférent (Ductus deferens) s'étend de la queue de l'épididyme au canal éjaculateur (Ductus ejaculatorius) par l'intermédiaire duquel il débouche dans l'urèthre (Urethra musculina).

Sa PORTION TESTICULAIRE longe médialement le bord épididymaire de la glande et $\mathrm{y}$ adhère intimement.

Dans sa PORTION INGUINALE OU FUNICULAIRE, le canal déférent forme avec les vaisseaux testiculaires et le Mesorchium proximal le cordon testiculaire ou cordon spermatique (Funiculus spermaticus).

Le cordon testiculaire est enfermé dans la gaine que constituent le crémaster externe, la tunique fibreuse et le feuillet pariétal de la séreuse. Le mésorchium proximal (Mesorchium proximale) qui le soutient s'insère caudalement sur la face interne de la fibreuse et cranialement porte à son bord libre les vaisseaux testiculaires. Dans le tissu conjonctif compris entre les deux lames séreuses qui le constituent, on peut noter la présence de fibres musculaires lisses qui forment le muscle crémaster interne ( $M$. cremaster internus). Le canal déférent est porté par un petit méso propre (Mesoductus deferens) qui se détache de la face médiale du Mésorchium proximal. La portion de ce dernier, qui est comprise entre le Mesoductus deferens et le feuillet pariétal de la séreuse, porte le nom de Mesofuniculus.

La PORTION ABDOMINO-PELVIENNE du canal déférent commence à l'anneau vaginal. Soutenu par le méso-déférentiel, le canal pénètre dans la cavité pelvienne et se place sur la face dorsale de la vessie. Il se dispose alors parallèlement et au contact de son homologue du côté opposé auquel l'unit cranialement le méso-interdéférentiel.

Dans sa PORTION SUPRA-VESICALE, le canal déférent se dilate pour former le renflement pelvien ou ampoule du canal déférent (Ampulla ductus deferentis).

La PAROI DU CANAL DEFERENT est épaisse. Elle comprend trois couches: une adventice (Tunica adventitia), qui en réalité est une sous-séreuse, une musculeuse (Tunica muscularis) très épaisse et une muqueuse (Tunica mucosa) marquée de plis longitudinaux. Son épithélium comprend, comme celui du canal épididymaire, une couche de cellules basales disséminées et une couche continue et unistratifiée de cellules cylindriques et ciliées. 


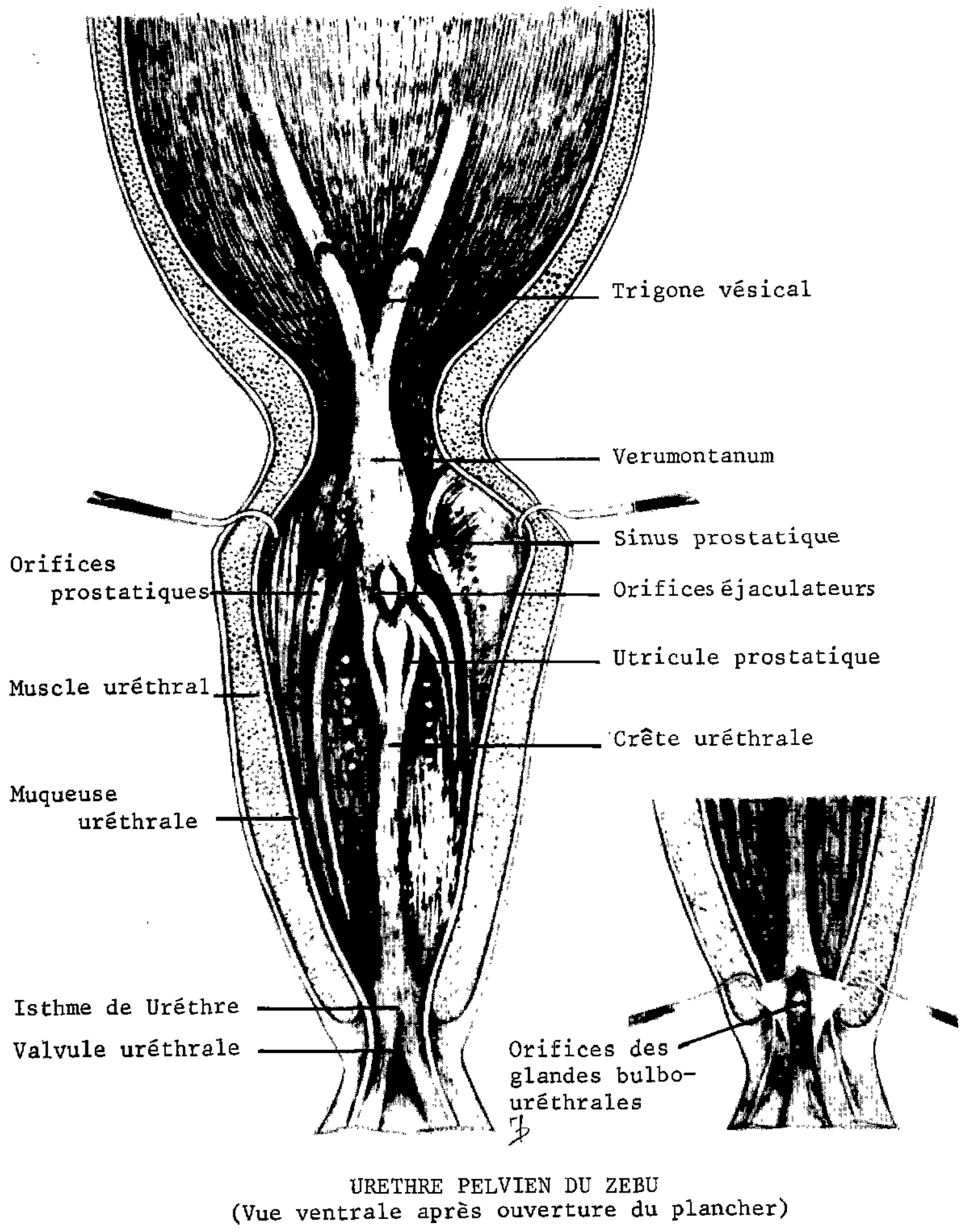


$\mathrm{Au}$ niveau du renflement ampullaire et à son voisinage, la muqueuse déférentielle délègue dans la sous-muqueuse des diverticules ramifiés (Diverticula ampullae) limités par une assise cellulaire cylindrique basse, ciliée et glandulaire.

\section{I.A.2.c) Les vésicules séminales}

Les vésicules séminales (Glandula vesicularis) sont deux formations glandulaires, allongées et d'aspect bosselé. Chacune est constituée comme chez Bos taurus d'un très long tube circonvolutionné.

Structuralement, le tube séminal est formé d'une adventice (Tunica adventitia) épaisse, d'une musculeuse très discrète (Tunica muscularis) et d'une muqueuse (Tunica mucosa) dont l'épithélium comprend une couche de cellules basales et une assise superficielle et simple de cellules glandulaires non ciliées.

\section{I.A.2.d) Le canal éjaculateur}

Le canal éjaculateur (Ductus ejaculatorius) très court, résulte de l'union du canal déférent et du canal excréteur de la vésicule séminale. Il débouche au plafond de l'urèthre pelvien au niveau de l'orifice éjaculateur (Ostium ejaculatorium).

\section{I.A.2.e) L'utricule prostatique}

L'utricule prostatique (Uterus musculinus) est habituellement absent chez le zébu. Sa portion caudale peut persister. Elle se présente alors sous l'aspect de deux petites invaginations du plafond de l'urèthre, situés en arrière du verumontanum.

\section{I.A.2.f) L'urèthre pelvien}

L'URETHRE PELVIEN (Urethra musculina - pars pelvina) est long et de calibre relativement faible. La crête uréthrale (Crista urethralis) et le verumontanum (Colliculus seminalis) sont peu développés. Les orifices éjaculateurs (Ostium ejaculatorium) sont larges. Dans les sinus prostatiques (Sinus prostaticus) situés de part et d'autre du verumontanum débouchent les canaux excréteurs de la prostate (Ductuli prostatici). Plus caudalement au niveau de l'isthme de l'urèthre (Isthmus urethrae), on peut observer les orifices nombreux et fins des canaux excréteurs des glandes bulbo-uréthrales (Ductus glandula bulbourethralis) qui débouchent sous une formation valvulaire.

La MUQUEUSE DE L'URETHRE PELVIEN (Tunica mucosa) est rougeâtre et plissée longitudinalement. Son chorion renferme des glandes uréthrales (Glandulae urethrales) de type racémeux. Les formations vasculaires d'aspect érectile (Stratum cavernosum) sont très rares dans la paroi de l'urèthre pelvien du zébu.

Le MUSCLE URETHRAL (Tunica muscularis) entoure le tube uréthral ventralement et latéralement. Il est d'autant plus épais qu'on se rapproche du bord ventral de l'organe. Il est recouvert d'une aponévrose mince ventralement et très épaisse dorsalement.

\section{I.A.2.g) La prostate}

La prostate (Prostata) est peu développée.

Sa portion diffuse (Pars disseminata prostatae), située sous le muscle uréthral, est discrète.

Sa portion condensée ou corps prostatique (Corpus prostatae), est réđuite. Elle se présente sous l'aspect d'une petite masse jaunâtre située sur la face dorsale de la portion craniale de l'urèthre.

Structuralement, la prostate du zébu est une glande tubulaire ramifiée dont les éléments se regroupent en lobules intimement intriqués les uns dans les autres. 


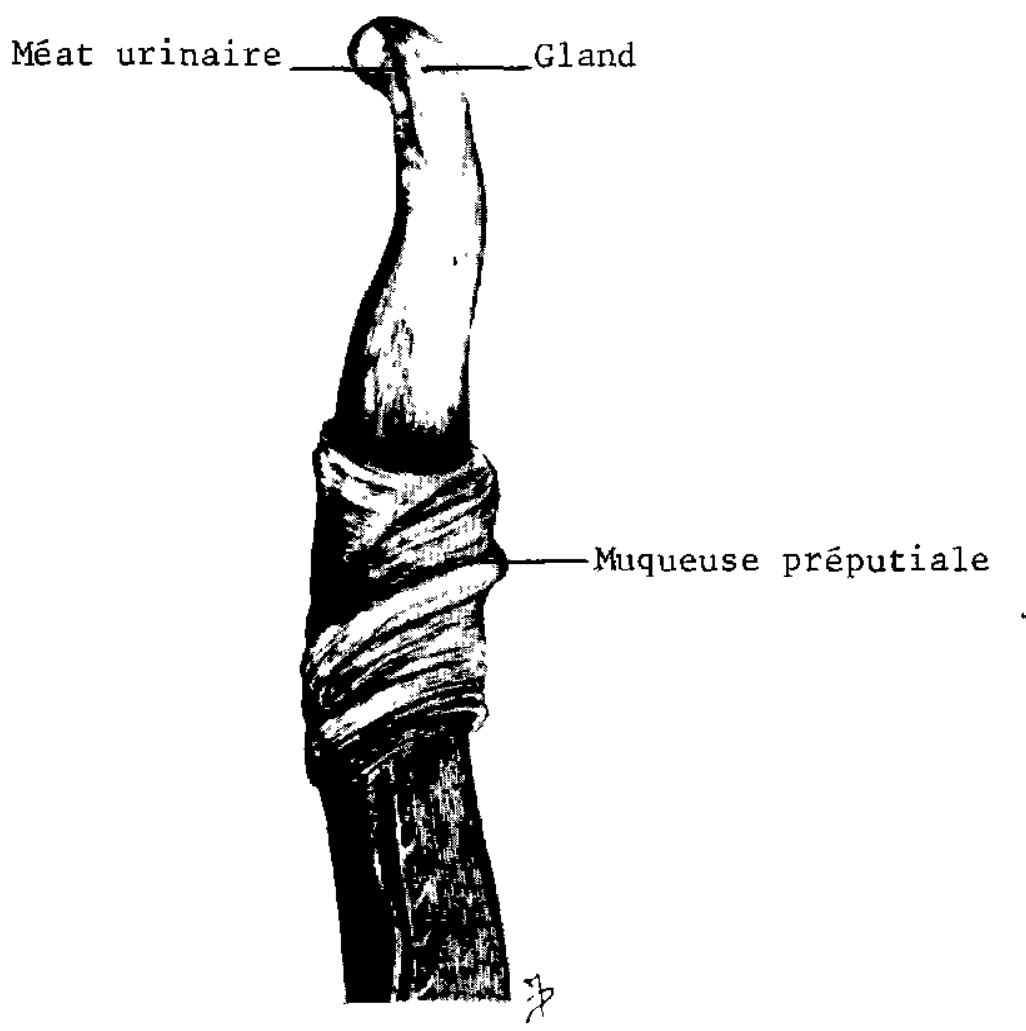

PORTION LIBRE DE LA VERGE DU ZEBU (Vue dorsale)

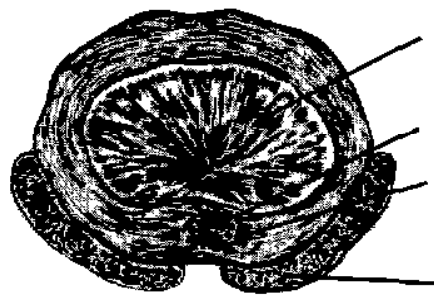

Corps caverneux

Urèthre

Corps spongieux de

1 'urèthre

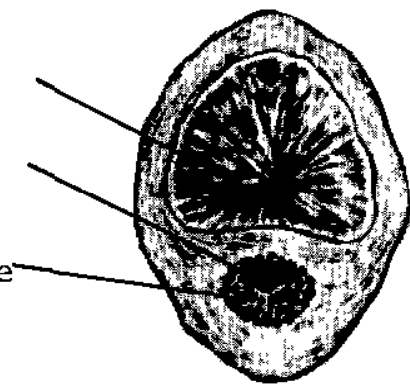

COUPES TRANSVERSALES DE LA VERGE
A) - En région caudale
B) - En région craniale 


\section{I.A.2.h) La glande bulbo-uréthrale (Glandula bulbourethralis)}

Les glandes bulbo-uréthrales se présentent sous l'aspect de deux corps arrondis situés sur la face dorsale de la portion terminale de l'urèthre pelvien. Elles sont recouvertes d'une couche de tissu fibreux dense et d'une couche musculaire épaisse dépendant du muscle bulbo-caverneux.

Structuralement les glandes bulbo-uréthrales sont du type tubulo-acineux. Le massif cellulaire qu'elles constituent est découpé en lobule par des cloisons issues de la couche fibreuse périphérique.

\section{I.A.2.i) Les culs-de-sac péritonéaux de la cavité pelvienne du taureau}

Le péritoine ne tapisse pas complètement les parois du bassin et les organes pelviens. Avant d'atteindre le fond de la cavité pelvienne, il se réfléchit des parois aux viscères en formant des culs-de-sac. Il résulte de cette particularité que les organes pelviens présentent une portion craniale recouverte par la séreuse péritonéale et une portion caudale sans rapport avec elle.

Le cul-de-sac recto-génital (Excavatio rectogenitalis) est très profond. Il s'engage très loin vers l'arrière entre le rectum et l'urèthre pelvien et se termine à $1 \mathrm{~cm}$ seulement du périné. (Perineum).

Le cul-de-sac vésico-génital ou vésico-déférentiel (Excavatio vesicogenitalis) n'atteint pas le fond du sinus formé par ces organes. Cette particularité fait que, si les faces dorsales des canaux déférents et des vésicules séminales sont entièrement tapissées par la séreuse, leurs faces ventrales répondent à la portion rétropéritonéale du bassin (Spatium retroperitoneale) dans leurs parties les plus caudales.

Le cul-de-sac pubo-vésical (Excavatio pubovesicalis) est peu profond. Il n'interfère que de 1,5 à $2 \mathrm{~cm}$ entre le plancher du bassin et la vessie, si bien que la face dorsale de l'urèthre pelvien est presque entièrement recouverte par la séreuse, tandis que sa face ventrale et la plus grande partie de celle de la vessie sont dans l'espace rétropéritonéal.

\section{I.A.3.) PORTION COPULATRICE DE L'APPAREIL GENITAL DU TAUREAU ZEBU}

\section{I.A.3.a) La verge ou pénis}

La VERGE ou PENIS (Penis) est longue, mais de diamètre réduit. Morphologiquement, elle se divise en 3 parties.

Sa racine (Radix penis) est subdivisée en 2 branches (Crus penis) fixées sur l'arcade ischiatique. Entre ces branches se trouve le bulbe de l'urèthre (Bulbus penis).

Sa portion moyenne ou corps de la verge (Corpus penis) est légèrement aplatie dorsoventralement en arrière, puis d'un côté à l'autre rostralement. Elle forme, comme chez Bos taurus, avant d'atteindre la région inguinale, une inflexion sigmoïde (Flexura sigmoidea penis) qui s'efface au moment de l'érection.

Sa portion craniale ou portion libre de la verge (Pars libera penis) est recouverte par la muqueuse préputiale. Son extrémité est coiffée d'une formation érectile particulière : le gland (Glans penis). Celui-ci est peu développé, légèrement aplati d'un côté à l'autre et pointu. Il présente un sillon légèrement spiralé orienté latéro-ventralement à l'extrémité duquel s'ouvre le méat urinaire (Ostium urethrae externum).

Structuralement, la verge est constituée de 3 formations érectiles réunies autour de la portion penienne de l'urèthre (Pars spongiosa urcthrae) : le corps caverneux, le corps spongieux de l'urèthre et le corps spongieux du gland.

Le CORPS CAVERNEUX (Corpus cavernosum penis) est limité par une albuginée (Tunica albuginea corporum cavernosum) épaisse qui limite l'accroissement de son diamètre au moment 
de l'érection. Celle-ci se traduit donc surtout par une rigidification et une augmentation de longueur de l'organe, due à l'effacement de la courbure sigmoïde. L'urèthre pénien, entouré de son corps spongieux est situé à l'intérieur du corps caverneux, à proximité du bord ventral.

Le CORPS SPONGIEUX DE LURETHRE (Corpus spongiosum penis) forme un manchon autour de l'urèthre pénien. A son origine, entre les racines du corps caverneux, il est volumineux et constitue le bulbe uréthral (Bulbus penis).

Le CORPS SPONGIEUX DU GLAND (Corpus spongiosum glandis) peu développé, coiffe l'extrémité craniale du corps caverneux. Il est en continuité de substance avec le corps spongieux de l'urèthre.

Le MUSCLE BULBO-CAVERNEUX ( $M$. bulbospongiosus) est court. I1 ne recouvre que le bulbe de l'urèthre.

Le MUSCLE ISCHIO-CAVERNEUX ( $M$. Ischiocavernosus) recouvre ventralement les racines de la verge sur lesquelles il s'insère.

Le MUSCLE RETRACTEUR DE LA VERGE ( $M$. retractor penis) passe entre les racines de la verge, longe le bord ventral de la courbure sigmoïde puis se fixe au bord ventral du pénis pour se terminer à la limite caudale de la portion libre.

Les LIGAMENTS SUSPENSEURS DU PENIS (Lig. suspensorium penis), très courts unissent la symphyse ischiale à l'extrémité postérieure du dos de la verge (Dorsum penis).

\section{I.A.3.b) Le fourreau}

Le fourreau (Preputium) est long et étroit. Son orifice (Ostium preputiale) entouré d'une touffe de longs poils est étroit. Il est placé très en avant, presque au niveau de l'ombilic, à distance de la paroi abdominale, à laquelle il est appendu ainsi que le fourreau par un long repli cutané.

Les muscles protracteur ( $M$. preputialis cranialis) et rétracteur ( $M$. preputialis caudalis) du fourreau sont bien développés.

La muqueuse préputiale rosâtre est plissée longitudinalement. Son chorion renferme des glandes tubulaires et des formations lymphoïdes diffuses.

\section{I.B.) Caractéristiques morphologiques du tractus génital de la femelle}

Dans ses grandes lignes, le tractus génital femelle de Bos indicus ressemble à celui de Bos taurus. II s'en distingue cependant par certaines particularités.

\section{I.B.1.) PORTION GLANDULAIRE : LES OVAIRES}

\section{I.B.1.a) Situation; conformation extérieure; rapports}

Comme ceux de Bos taurus, les ovaires (Ovaria) de Bos indicus subissent une migration pendant leur développement. A la fin de celle-ci, ils sont situés de part et d'autre de l'entrée du bassin, à proximité de l'insertion du muscle petit psoas sur le col de l'ilium. La migration ventro-craniale qu'ils subissent à chaque gestation, par relâchement du ligament large (Lig. latum uteri) et du ligament suspenseur de l'ovaire ( $L i g$. suspensorium ovarii) est beaucoup moins importante chez le zébu que chez les bovins d'Europe. Cette particularité doit vraisemblablement être rapportée à l'âge tardif auquel s'effectue habituellement le $1^{\text {er }}$ vêlage et la période relativement longue qui sépare deux gestations consécutives.

Comme celui de Bos taurus, l'ovaire de Bos indicus se présente souvent sous la forme d'une amande aplatie latéro-médialement. Son gros pôle est orienté vers l'infundibulum tubaire (Extremitas tubaria). Son petit pôle vers l'extrémité de la corne utérine (Extremitas uterina). 
Souvent, cependant, les ovaires du zébu sont ovalaires, à bords dorsal (Margo mesovaricum) et ventral (Margo liber) presque parallèles et pôles subégaux.

Par sa taille, l'ovaire du zébu est nettement plus petit que celui des bovins d'Europe. Il mesure en moyenne 2,5 à $3 \mathrm{~cm}$ de longueur, 1,2 à $1,8 \mathrm{~cm}$ de largeur et 0,8 à $1,3 \mathrm{~cm}$ d'épaisseur.

En raison de la migration subie par la gonade, le ligament suspenseur de l'ovaire (Lig. suspensorium ovarii) et la portion la plus craniale du ligament large (Mesovarium) sont relativement longs.

La bourse ovarique (Bursa ovarica) est largement ouverte. Son orientation et celle de la glande qu'elle renferme sont inversées par rapport à celles de la plupart des autres espèces. En raison du reploiement que subit médialement et caudalement le mésovarium par rapport au reste du ligament large, le pôle cranial de l'ovaire ou extrémité tubaire devient caudal, le mésovarium distal sur lequel se fixe la glande prend une position latérale et le mésosalpinx long et à bord libre convexe devient médial.

L'ovaire est fixé au mésovarium distal par son bord dorsal. Son extrémité tubaire est fixée au pavillon de l'oviducte par le ligament tubo-ovarien (Fimbria ovarica). Son extrémité utérine est unie à l'utérus par le ligament utéro-ovarien ou ligament propre de l'ovaire ( $\mathrm{Lig}$. ovarii proprium).

\section{I.B.1.b) Structure de l'ovaire}

1. L'EPITHELIUM OVARIEN recouvre presque entièrement l'ovaire. Il n'est absent qu'au niveau du bord dorsal par lequel l'ovaire adhère au mésovarium distal et au niveau du hile (Hilus ovarii), le plus souvent localisé vers l'extrémité tubaire.

2. La ZONE CORTICALE (Cortex ovarii) est mince.

3. La ZONE MEDULLAIRE est caractérisée par le fait que ses deux portions, la zone parenchymateuse (Zona parenchymatosa) et la zone vasculaire (Zona vasculosa) ont une limite peu nette en raison de l'extension fréquente des gros vaisseaux péri-hilaires dans le stroma ovarien.

4. Les ORGANITES EVOLUTIFS peuvent être, topographiquement et fonctionnellement, divisés en trois groupes.

- Les follicules primordiaux et primaires (Folliculi ovarici primarii) sont localisés dans la zone corticale. Ils se présentent habituellement sous la forme d'une couche unie ou bistratifiée, continue, ou interrompue par l'interférence d'organites plus volumineux. Ils sont relativement peu nombreux et avec l'âge leur nombre diminue rapidement. Vers la $10^{\mathrm{k}}$ ou la $11^{\mathrm{e}}$ année, ils deviennent très rares.

- Les follicules cavitaires (Folliculi ovarici vesiculosi) dérivent des précédents; lorsqu'ils sont de taille petite ou moyenne, ils se localisent dans la zone parenchymateuse et y occupent une position d'autant plus profonde qu'ils sont plus volumineux. Plus nombreux chez les jeunes, à partir de la puberté, que chez les vieux, ils subissent aussi des variations numériques avec le cycle œstral.

- Les gros follicules cavitaires, les follicules mûrs et les follicules déhiscents ne sont visibles qu'au moment du procstrus et de l'œstrus. Toujours peu nombreux (1 à 4), ils proviennent du stock des follicules cavitaires petits et moyens. En subissant la maturation, ils deviennent plus superficiels au fur et à mesure qu'ils grossissent et finissent, au stade follicule mûr, par s'accoler à l'épithélium ovarien. Un seul follicule atteint habituellement la déhiscence. Lorsque d'autres ont commencé la maturation avec lui, ils involuent plus ou moins précocement.

5. Les ORGANITES INVOLUTIFS dérivent en grande majorité du groupe des follicules cavitaires petits et moyens, qui, schématiquement, de façon cyclique se reconstitue donc à partir du stock des follicules primordiaux et primaires et s'épuise par involution et maturation folliculaire. 


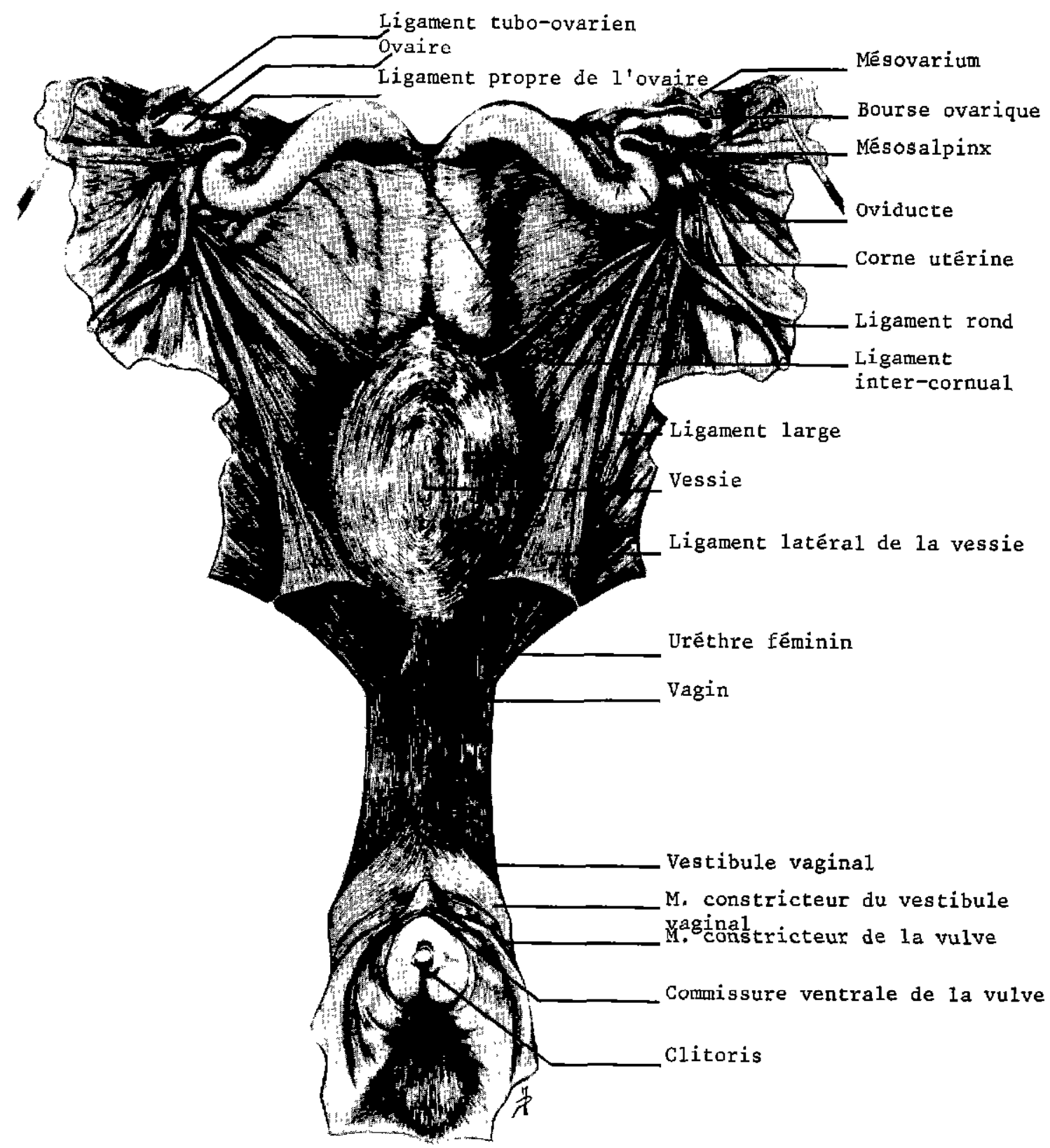

TRACTUS GENITAL DE LA FEMELLE ZEBU

(Conformation extérieure vue ventrale) 
- Les organites involutifs issus des follicules cavitaires petits et moyens que nous avons observés sont en grande majorité du type follicule atrétique, donc doués d'activité hormonale.

- Les follicules kystiques ou kystoïdes sont rares. Ils dérivent des grands follicules cavitaires qui ont subi un début de maturation en même temps que le futur follicule déhiscent.

- Les follicules involutifs dérivés des follicules primaires et primordiaux sont très rares. Nous ne les avons observés qu'une fois sur un sujet au deuxième mois de la gestation qui présentait une involution massive et bilatérale de la totalité de son stock folliculaire.

6. Les CORPS GESTATIFS ET PROGESTATIFS ne présentent pas de différences par rapport à ceux de Bos taurus. Fréquemment, ils revêtent l'aspect caractéristique dit " en bouchon de champagne ». Souvent très volumineux, les corps gestatifs envahissent l'ovaire en refoulant les organites et le stroma qui n'occupent plus alors sur les coupes histologiques qu'une étroite zone en croissant centrée sur le corps jaune.

\section{I.B.2.) PORTION TUBULAIRE DU TRACTUS GENITAL DE LA FEMELLE}

\section{I.B.2.a) L'oviducte}

L'oviducte (Tuba uterina) mesure 10 à $15 \mathrm{~cm}$ de longueur. Il a un trajet très sinueux. Il est compris entre les 2 lames séreuses du mésosalpinx qu'il parcourt à distance du bord libre selon un trajet courbe à concavité dorsale. Son diamètre est d'autant plus petit qu'il se rapproche de l'ovaire.

Le pavillon de l'oviducte (Infundibulum tubae uterinae) est pourvu de franges (Fimbriae tubae) dont l'une (Fimbria ovarica) est plus longue que les autres et adhère à l'extrémité tubaire de l'ovaire.

La jonction tubo-utérine (Pars uterina) se fait progressivement, par augmentation régulière du diamètre.

La muqueuse tubaire (Tunica mucosa) est garnie de plis longitudinaux. Son épithélium est composé de cellules ciliées et de cellules glandulaires.

\section{I.B.2.b) L'utérus}

L'utérus (Uterus) est de type bicorne (Uterus bicornis).

\section{CONFORMATION EXTERIEURE}

Le corps utérin (Corpus uteri) très court est presque virtuel.

Les cornes utérines (Cornua uteri) sont très longues. Caudalement, elles sont placées côte à côte et leurs faces médiales sont unies par du tissu conjonctif tandis qu'un feuillet séreux commun les recouvre. Cranialement, elles divergent et sont contournées en $\mathrm{S}$ latéro-ventralement, puis médio-caudalement. Dans l'angle de divergence des 2 cornes, un repli séreux simple apparaît. Il constitue le ligament intercornual (Lig. intercornuale).

Le col utérin (Cervix uteri) est long et volumineux. Sa consistance est ferme.

\section{CONFORMATION INTERIEURE}

La cavité utérine (Cavum uteri) est pratiquement virtuelle, chez la femelle non gestante.

La muqueuse du corps et des cornes est garnie de reliefs arrondis tassés les uns contre les autres : les caroncules (Carunculae) sur lesquelles se fixent les cotylédons fataux (Cotyledon) pendant la gestation. En raison de la disposition parallèle de la portion caudale des deux cornes, les parois médiales de ces dernières forment intérieurement un éperon ou vélum utérin (Velum uteri) qui vient au contact de l'orifice interne du col (Ostium uteri internum) et rend virtuelle la cavité du corps utérin.

Le canal cervical (Canalis cervicis uteri) est très long et de diamètre réduit. 


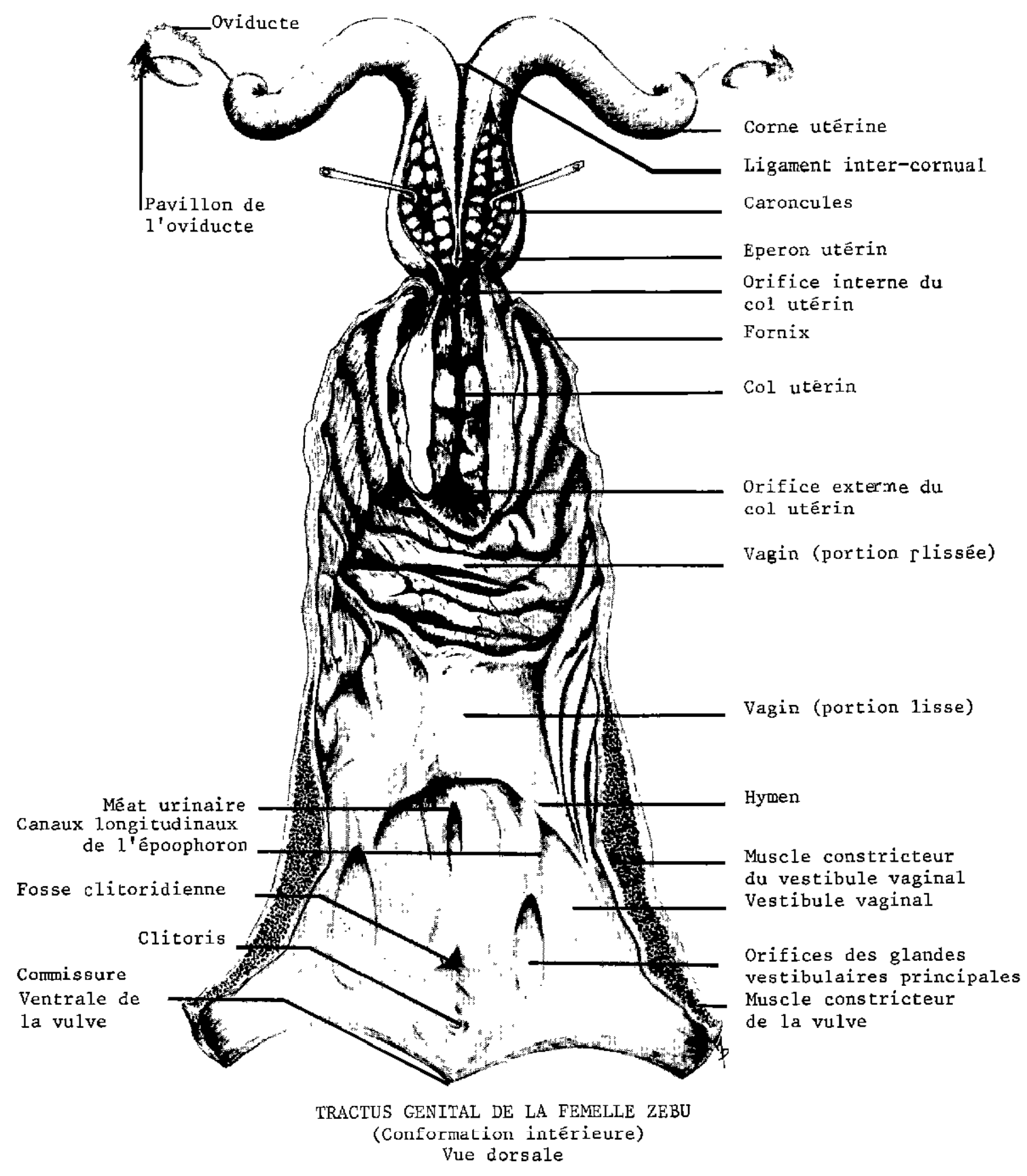


Son orifice interne (Ostium uterii internum) est étroit. Son orifice externe (Ostium uteri externum) est large et infundibulaire.

Sa muqueuse (Endometrium) est marquée par 4 ou 5 forts reliefs circulaires et épais (Plicae circulares).

Le vagin interfère largement sur le col de sorte que la portion prévaginale (Portio prevaginalis) de ce dernier est presque nulle, tandis que sa portion vaginale (Portio vaginalis) est très longue.

\section{LES LIGAMENTS LARGES}

Les ligaments larges (Lig. latum uteri) maintiennent en place l'utérus à l'entrée du bassin.

Par son bord dorsal fixe, chacun d'eux s'insère cranialement à la région sous-lombaire. Caudalement, cette insertion s'effectue de plus en plus ventralement et latéralement de sorte que dans le bassin les ligaments larges se fixent sur les parois latérales de la cavité pelvienne.

- Le bord ventral libre de chaque ligament large se fixe sur le bord ventral (Margo mesometricus) de la corne utérine correspondante puis à la limite des faces ventrale et latérale du corps et du col utérin.

- La face externe du ligament large présente un ligament rond (Lig. teres uteri) bien développé. Celui-ci débute à proximité de l'ovaire par un appendice arrondi et flottant. Il se termine caudalement au niveau de l'anneau inguinal profond. Les ligaments latéraux de la vessie ( $L i g$. vesicae laterale) se détachent de la face externe des ligaments larges.

\section{STRUCTURE}

La muqueuse utérine (Tunica Mucosa) ou endomètre (Endometrium) se compose d'un épithélium cylindrique simple à cellules ciliées et sécrétoires et d'un chorion riche en glandes en tube. Epithélium et glandes présentent des variations cycliques.

Le muscle utérin (Tunica muscularis) ou myomètre (Myometrium) est bien développé. Il subit aussi des variations quantitatives avec le cycle cestral.

La séreuse (Tunica serosa) ou périmètre (Perimetrium) tapisse la presque totalité de l'utérus. Seule la face ventrale du col répond à l'espace rétropéritonéal.

\section{I.B.3.) PORTION COPULATRICE DU TRACTUS GENITAL FEMELLE}

\section{I.B.3.a) Le vagin}

Le VAGIN (Vagina) est volumineux. Il est aplati dorso-ventralement. Au niveau du Fornix vaginae, il interfère très largement sur le col qu'il enveloppe presque complètement si bien qu'extérieurement son extrémité craniale apparaît sous l'aspect d'un gros bourrelet circulaire dans lequel semblent s'engager côte à côte les cornes utérines.

Intérieurement, le vagin peut être divisé en trois parties subégales : une portion caudale tapissée par une muqueuse lisse; une portion moyenne caractérisée par de gros replis circulaires; une portion craniale en cul-de-sac circulaire profend. Elle correspond au fornix (Fornix vaginae) et présente aussi une muqueuse garnie de gros bourrelets circulaires qui se réfléchissent sur le revers vaginal du col.

L'HYMEN (Hymen) est peu développé.

\section{I.B.3.b) Le vestibule vaginal}

Le vestibule vaginal (Vestibulum vaginae) est très long. Il mesure à peu près les $2 / 3$ de la longueur du vagin.

Il est tapissé d'une muqueuse lisse. Sur son plancher, le méat urinaire (Ostium urethrae externum) s'ouvre largement à l'extrémité craniale d'une crête uréthrale (Crista urethralis) peu 


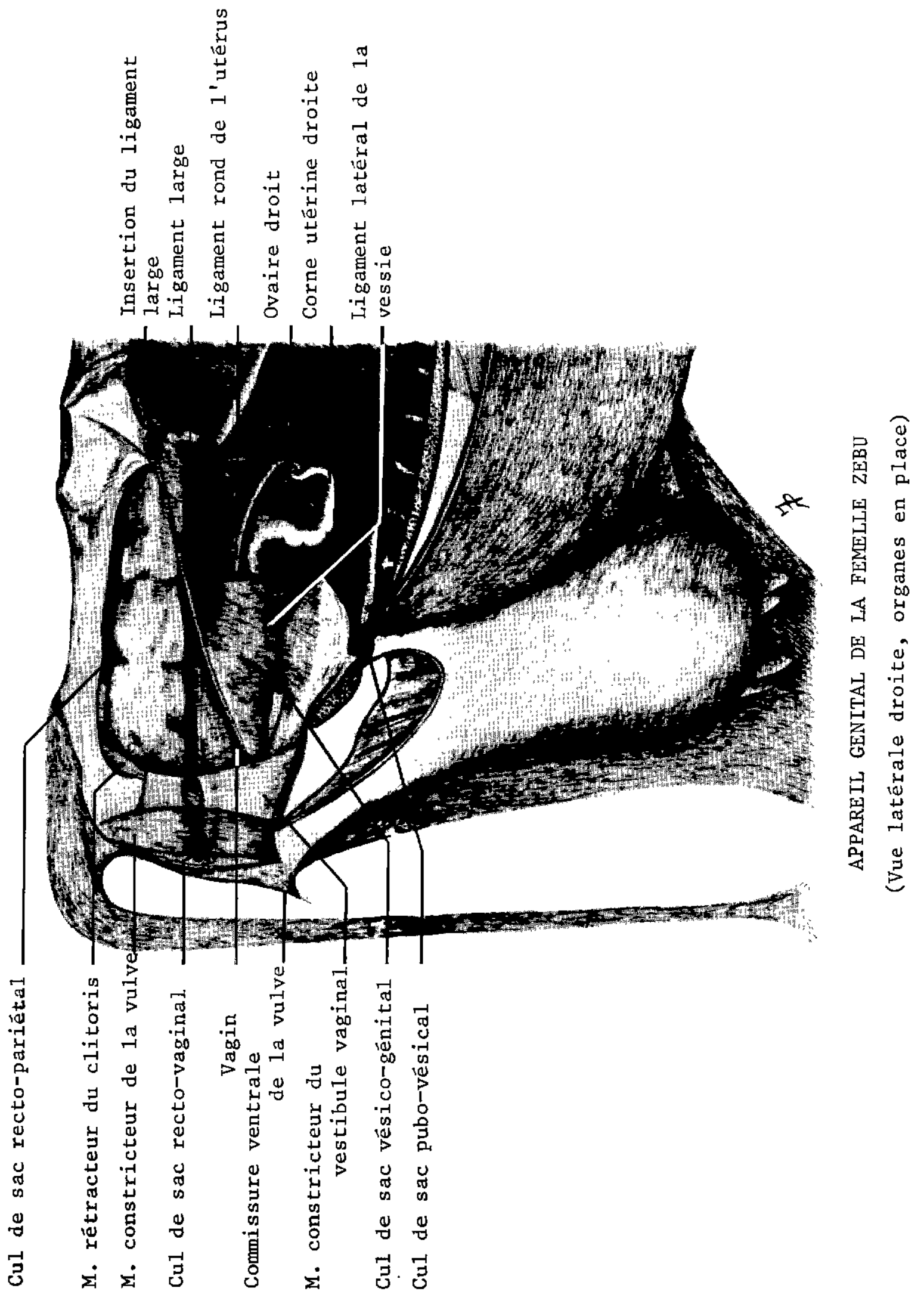


marquée. A proximité du méat, le plancher de l'urèthre féminin (Urethra feminina) du zébu présente comme celui des taurins un diverticule ventral (Diverticulum sub-urethrale) dont le plafond a l'aspect d'une valvule.

Latéralement et caudalement au méat se trouvent les orifices des canaux longitudinaux de l'epoophoron (Ductus epoophori longitudinales) ou canaux de Gartner qui s'étendent cranialement dans l'épaisseur des parois vestibulaire et vaginale. Ils se terminent en cul-de-sac, à des niveaux variables mais sans jamais atteindre l'utérus.

Caudalement aux canaux de Gartner, dans la concavité de deux fosses ovales peu profondes débouchent, par de petits orifices, les canaux des glandes vestibulaires principales (Gl. vestibularis majores) ou glandes de Bartholin.

La paroi vestibulaire contient encore deux formations érectiles latérales : les bulbes vestibulaires (Bulbus vestibuli) et un muscle constricteur ( $M$. constrictor vestibuli).

\section{I.B.3.c) La vulve}

La vulve (Pudendum femininum-Vulva) est formée par deux lèvres latérales (Labium pudendi) limitée par deux commissures.

La commissure dorsale (Commissura labiorum dorsalis) est étroite.

La commissure ventrale (Commissura labiorum ventralis) est saillante. A son revers interne on observe, dans la fosse clitoridienne (Fossa clitoridis), l'extrémité libre du clitoris (Glans clitoridis) recouverte d'un repli muqueux (Preputium clitoridis).

Des formations musculaires sont associées à la vulve : le muscle constricteur de la vulve ( $M$. constrictor vulvae) et le muscle nétracteur du clitoris ( $M$. retractor clitoridis) dont les deux faisceaux croisent latéralement la paroi vulvaire.

\section{I.B.3.d) Les culs-de-sac péritonéaux de la cavité pelvienne de la femelle zébu}

Le cul-de-sac recto-génital est plus profond encore que chez le mâle. Il atteint le périné.

Le cul-de-sac vésico-génital et le cul-de-sac pubo-vésical sont comparables à ceux du mâle. La presque totalité de l'urèthre et de la paroi ventrale du vagin se trouvent de ce fait dans l'espace rétropéritonéal.

\section{BASES FONCTIONNELLES DE LA VIE GENITALE DU ZEBU}

$\mathrm{Si}$, concernant la morphologie de l'appareil génital du zébu, de significatives particularités peuvent être notées, du point de vue fonctionnel, la vie génitale de Bos indicus diffère aussi sensiblement, de celle de Bos taurus.

En ce domaine, les travaux qui ont été réalisés relèvent de deux disciplines complémentaires. Les plus anciens et les plus nombreux sont d'ordre zootechnique. Ils sont utilement complétés par des recherches histo-physiologıques entreprises depuis 4 ans à l'Ecole Inter-Etats des Sciences et Médecine Vétérinaires de Dakar.

Nous exposerons successivement les résultats obtenus dans les 2 catégories de recherche.

\section{II.A.) Recherche zootechnique}

Bien que la reproduction du zébu soit, dans les conditions naturelles, soumises à un rythme annuel très net, aucune des publications que nous avons consultées ne se prononce sur la nature, saisonnière ou continue, du cycle génital de cette espèce. Il convient encore de noter que, mis à part quelques travaux relatifs à l'insémination artificielle, seule la femelle a pratiquement retenu l'attention des zootechniciens. 


\section{II.A.1.) DUREE DU CYCLE GSTRAL}

La durée du cycle ostral du zébu varie généralement entre 19 et 23 jours:

- DONALDSON (1962) .

19 à 22 jours

- BHATTACHARYA et Collab. (1964 aux Indes) . $21,16 \pm 2,26$ jours

- ANDERSON (1964 au Kenya) 23,03 jours

- ASDELL (1946) aux U.S.A. 23,03 jours

Des observations effectuées par DENIS (1971) à Dahra, il résulte que le cycle est un peu plus court chez les génisses $(21,11 \pm 0,50$ jours $)$ que chez les vaches adultes $(21,8$ jours).

$\mathrm{Au}$ total, la durée moyenne du cycle est proche de celle de Bos taurus, estimée habituellement entre 20 et 23 jours.

\section{II.A.2.) L'ASTRUS}

Tous les auteurs s'accordent pour considérer comme très discret et difficilement observable l'œstrus de la vache zébu. Il se produit souvent la nuit: 40 fois sur 100 selon ROLLINSON (1965) au Kenya; 31,17 fois sur 100 selon BAKER (1967) en Australie.

Certains auteurs l'estiment même fréquemment invisible en raison de l'absence de signes externes et le classent ainsi dans la catégorie des Oestrus silent heat (Estrus à chaleur silencieuse) caractérisés par des phénomènes cycliques normaux du tractus génital sans manifestations extérieures.

Les anglo-saxons ont coutume de diviser l'œstrus en 3 périodes. Au cours de la première, la femelle attire le mâle mais refuse l'accouplement. Pendant la deuxième, elle accepte la saillie. Pendant la troisième, elle refuse à nouveau le coït, mais attire toujours le mâle.

La période totale d'attirance du mâle semble très variable. Elle peut aller de 7,47 à 43,27 heures, selon ROLLINSON (1955) en Uganda.

Les chiffres les plus fréquemment avancés sont compris entre 13 et 23 heures:

- 13,3 heures pour CLAMCHOY (1952) aux Philippines;

- 14 à 16 heures pour DENIS (1971) à Dahra;

- 19,80 $\pm 2,98$ heures selon BAKER (1967) en Australie;

- 21,07 heures pour BISSOCHOP (1941) en Afrique du Sud.

La période d'acceptation du mâle semble aussi de durée très variable. Elle peut aller de 12 minutes à 36 heures, selon ROLLINSON (1955) en Uganda. Les chiffres les plus couramment avancés varient entre 4 et 8 heures :

- 4,78 heures pour ANDERSON (1944) au Kenya;

- 5 à 6 heures selon DENIS (1971) à Dahra;

— 7,88 heures pour BISSOCHOP (1941) en Afrique du Sud.

Par rapport à celui de Bos taurus qui se répète régulièrement et dont la durée est estimée entre 18 à 20 heures, l'cestrus de Bos indicus est donc caractérisé par sa durée variable, la fréquente brièveté de la période d'acceptation du mâle, la discrétion habituelle des manifestations extérieures et l'existence d'Oestrus silent heat.

\section{II.A.3.) AGES DE LA PUBERTE, DE LA PREMIERE SAILLIE FECONDE ET DU PREMIER VELAGE}

Les PREMIERES CHALEURS sont souvent tardives. Elles apparaissent, selon MAHADEVAN (1966), entre le $30^{\mathrm{e}}$ et le $42^{\mathrm{e}}$ mois. Cependant, PAGOT (1943 et 1951-52), au Niger, note qu'elles peuvent se manifester entre le $18^{\mathrm{e}}$ et le $24^{\mathrm{e}}$ mois. 
La PREMIERE SAILLIE FECONDE s'effectue souvent beaucoup plus tard que la puberté, si bien que le premier vêlage se produit à un âge qui varie entre 3 et 5 ans :

- 4 à 5 ans au Sénégal (REDON, 1962);

- entre 3 et 5 ans en Mauritanie (PRIGENT et Collab., 1942);

- vers 3 ans et 5 mois au Niger (PAGOT, 1943 et 1951-52);

- vers 3 ans 1/2 en Ouganda (MAHADEVAN et MARPLES, 1961);

- entre 3 ans et 5 mois (NATTO et Collab., 1965) et 3 ans et 10 mois en moyenne (HILL, 1967), au Brésil;

- vers 4 ans $1 / 2$ - 5 ans au Ruanda (HERIN, 1952).

Ces chiffres, bien que concernant des zones climatiques variables, des races diverses et des troupeaux soumis à des méthodes d'élevage différentes, montrent que la puberté et le premier vêlage sont beaucoup plus tardifs chez Bos indicus que chez Bos taurus pour lequel la puberté s'effectue souvent avant un an et le premier vêlage avant 3 ans.

\section{II.A.4.) DUREE DE LA GESTATION}

La durée de la gestation est un peu inférieure à 300 jours (REDON, 1962). Selon MAHADEVAN (1966), elle varie de 280 à 290 jours.

Les plus grandes variations ont été observées par CHOWDHURI et SINHA, 1951, sur la race Tharparkar aux Indes; selon ces auteurs, la durée de la gestation est de $287 \pm 34$ jours.

Pour le zébu Gobra, DENIS (1971) à Dahra évalue la durée de la gestation à 292 $\pm 4,9$ jours; il remarque qu'elle est différente selon que le produit est de sexe mâle ou de sexe femelle et rapporte les chiffres suivants : 291,8 jours pour les mâles et 292,9 jours pour les femelles.

SINGH et RAY (1961) sur la race Red-Sindhi confirment cette observation de l'influence du sexe du produit mais obtiennent des résultats opposés avec les chiffres suivants : 287 $\pm 0,5$ jours pour les mâles et $285 \pm 0,5$ jours pour les femelles.

PRABHU (1961) insiste sur le fait que la race est aussi responsable d'importantes variations.

En règle générale, on peut donc admettre que la gestation est un peu plus longue chez Bos indicus que chez Bos taurus.

Le croisement donne des produits dont la durée de gestation est intermédiaire. JOUBERT et BONSMA (1959), en Afrique du Sud, relèvent les chiffres suivants : la gestation dure 287,3 $\pm 1,04$ jours pour la race Hereford élevée localement, $295 \pm 0,32$ jours pour le zébu Africander et $291,1 \pm 0,93$ jours pour le métis.

\section{II.A.5.) INTERVALLE ENTRE DEUX VELAGES}

L'intervalle entre deux vêlages est souvent long. Il varie de 382 jours, soit un peu plus de 12 mois 1/2 sur les races Nandi, Boran et Jidde (GALUKENDI et Collab., 1962; MAHADEVAN et HUTCHISON, 1964; Stations Expérimentales du Kenya et du Tanganyika) à 2 ans pour la race Gobra (REDON, 1962, au Sénégal).

Les chiffres moyens oscillent autour de 420 jours :

- 388 jours (MAHADEVAN et Collab., 1962) pour la race Sahiwal au Kenya;

- 395 jours (N.D.R.I., 1961) pour la race Kénana au Soudan;

- 420 jours (PLASS et Collab., 1968) en Floride;

- 437 jours (N.D.R.I., 1961) pour la race Red Sindhi aux Indes;

- 480 jours (JOSHI et PHILLIPS, 1953) pour le bétail des Indes et du Pakistan.

Pour une meilleure compréhension des faits, l'intervalle qui sépare deux vêlages consécutifs 


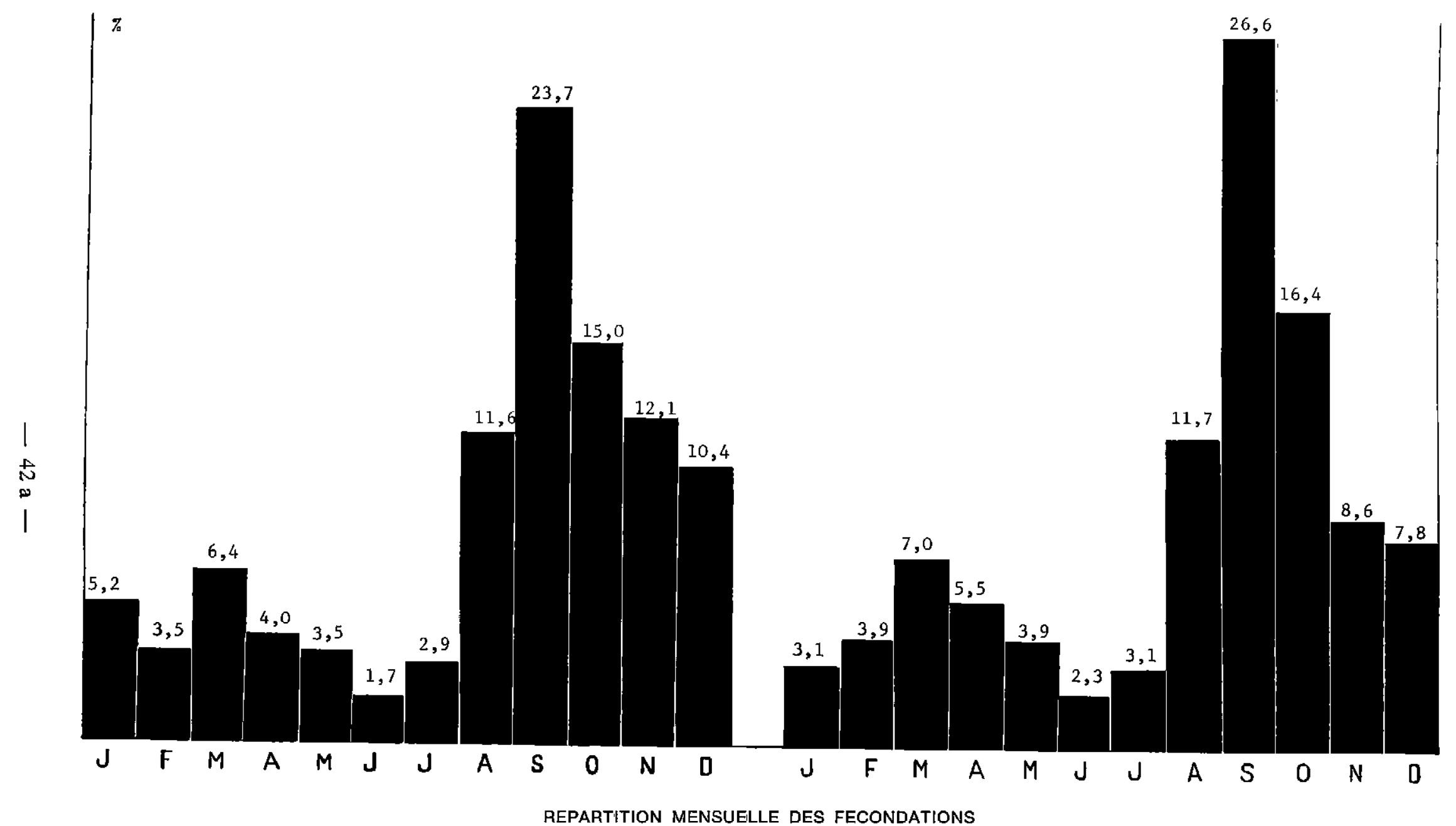

A) Pour la totalité des observations (173 femelles)
Moyenne mensuelle théorique : 8,3 p. 100.

B) Entre le 1er janvier et le 31 décembre 1970 (128 femelles)

(D'après CUQ, FERNEY et VAN CRAEYNEST in Rev. Méd. vét., 1974, 125 (2). 
peut être divisé en trois périodes. La première correspond à l'écart qui sépare la mise-bas du retour des chaleurs, la seconde au temps qui s'écoule entre le premier cestrus et la fécondation et la dernière, déjà étudiée, à la durée de la gestation.

Le RETOUR DES CHALEURS s'effectue entre le $83^{e}$ et le $161^{\text {e }}$ jour après le part (RICHARD, 1946, au Nigéria). Il est cependant fréquemment plus précoce :

CURASSON (1948) l'observe au 55e jour.

PLASSE et Collab. (1968), en Floride, le situent à $65,3 \pm 1,31$ jours pour le zébu Brahma.

Le principal et classique facteur limitant du retour des chaleurs est la lactation. Chez le zébu, elle se prolonge pendant 10 mois environ.

Selon DENIS (1971), sur le zébu Pakistanais étudié à Dahra, elle dure 241,4 $\pm 7,4$ jours mais est souvent plus courte puisque seulement 47,2 p. 100 des lactations dépassent 6 mois et 26,3 p. 100,9 mois. Pendant cette période la production laitière toujours limitée croit les deux premiers mois puis diminue régulièrement de 8 p. 100 par mois pour chuter ensuite rapidement après le sevrage du veau.

Au total, la lactation semble peu influencer la date du retour des chaleurs qui, dans la plupart des cas, diffère peu de celle admise classiquement pour Bos taurus (21 à 80 jours). C'est dans la deuxième période de l'intervalle entre deux vêlages qu'il faut chercher les causes des importantes variations enregistrées.

La PERIODE QUI SEPARE LE 1er GSTRUS POST-PARTUM DE LA FECONDATION (Service-period des auteurs anglo-saxons) est sujette à d'importantes variations. MAHADEVAN (1966) estime qu'elle est comprise entre 85 et 450 jours.

PRASAD (1958) sur la race Tharparkar l'évalue en moyenne à 131 jours mais souligne que son coefficient de variations est de 161 p. 100.

Quelques facteurs qui font varier cette période ont été mis en évidence : le nombre des gestations antérieures, le nombre des saillies nécessaires pour obtenir la fécondation et la saison à laquelle s'effectue le rapprochement sexuel.

Les gestations antérieures semblent jouer un rôle favorable sur la "période de service ". Selon KOHLI et SURI (1960), sur la race Hariana sa durée moyenne est de 263 jours pour l'ensemble du troupeau; elle s'élève à 283 jours pour les vaches primipares.

Le nombre des saillies nécessaires pour obtenir la fécondation dépend de l'état fonctionnel de l'appareil génital des deux sexes. Il est étudié dans la seconde partie de ce chapitre. Nous nous bornerons donc à rapporter ici l'observation de KOHLI et Collab. (1961), selon laquelle 1,72 saillies sont en moyenne nécessaires pour assurer la fécondation avec des taureaux de race Hariana âgés de 2 à 5 ans, tandis que 2,02 saillies doivent être en moyenne effectuées par les sujets âgés de 9 à 13 ans.

La notion de saison de reproduction joue un rôle capital dans l'intervalle qui sépare deux vêlages consécutifs.

\section{II.A.6.) SAISONS DE REPRODUCTION ET RYTHME ANNUEL DES FECONDATIONS}

L'observation effectuée par PAGOT (1951-52) sur le zébu Azaouak met bien en évidence l'importance de la saison sur le rythme de reproduction. L'auteur constate qu'au Niger, les vaches du troupeau qu'il étudie se répartissent en deux groupes : celui pour lequel l'intervalle est de 10 à 18 mois (moyenne 14 mois) et celui pour lequel l'intervalle est de 20 à 27 mois (moyenne 24 mois). Le premier groupe représente 35 p. 100 de l'effectif et le second 65 p. 100.

La durée moyenne de la lactation étant pour l'ensemble des sujets de 293 jours, soit 
environ 10 mois, il ressort des données précédentes que pour le $1^{\text {er }}$ groupe 4 à 5 mois séparent la fin de la lactation du vêlage suivant, tandis que pour l'autre cet écart est de 14 mois.

Dans le premier cas, la fécondation s'est effectuée vers le $5^{\mathrm{e}}$ mois du post-partum, c'est-à-dire pendant la lactation et à une saison favorable. Dans le second, la fécondation s'est produite 15 mois après le premier vêlage, soit 5 mois au moins après la fin de la lactation. Cette latence, dans la plupart des cas, doit être rapportée à l'attente d'une période favorable à la reproduction.

PAGOT distingue ainsi 3 périodes dans l'année. Une période de forte fécondité qui correspond à la saison des pluies et dure de mai à septembre, une période de failble fécondité qui uure d'octobre à janvier et correspond au début de la saison sèche, une période de moyenne fécondité qui débute en février et se termine en mai.

Au Sénégal (DENIS, 1971), la période de forte fécondité commence en août et se termine en novembre. Elle correspond à la saison des pluies.

CUQ, FERNEY et VAN CRAEYNEST (1974), d'une étude statistique effectuée à l'abattoir de Dakar, sur 173 femelles pleines, concluent que le quart environ des fécondations de l'année s'effectuent en septembre, qu'entre août et octobre plus de la moitié des fécondations annuelles se produisent et que, si l'on inclut le mois de novembre dans cette période, c'est près des $2 / 3$ des fécondations annuelles qui s'effectuent. Le reste de l'année, le taux mensuel des fécondations est très bas. Il ne subit un très léger accroissement qu'en avril et mai.

Ce léger accroissement de la fin du premier trimestre est confirmé par l'observation de PAGOT au Niger, déjà signalée, et celle de PRIGENT et Collab. (1942) qui rapportent qu'en Mauritanie les pasteurs Peulh essayent de regrouper les saillies en octobre et novembre (fin de la saison des pluies) et en mars-avril.

Par le caractère nettement saisonnier de sa reproduction, le zébu diffère donc fortement du bovin d'Europe qui, malgré une certaine recrudescence de la vie génitale au printemps et un certain assoupissement hivernal pour les sujets en stabulation, n'en conserve pas moins la capacité de se reproduire toute l'année.

L'importance de la saison sur la répartition annuelle des fécondations est telle qu'une étude plus poussée des facteurs qui interviennent doit être effectuée.

\section{II.A.7.) FACTEURS SAISONNIERS CAPABLES D'INFLUENCER LA REPRODUCTION}

\section{II.A.7.a) L'alimentation}

Le rôle de l'alimentation, particulièrement abondante en saison des pluies, est clairement démontré par nombre d'observations.

Elle intervient de façon très nette sur l'œstrus en rendant plus visibles les manifestations extérieures des chaleurs. DENIS (1971) à Dahra note que sur un lot nourri et abreuvé à volonté « les chaleurs deviennent très visibles: la vulve est cdémaciée, la muqueuse vaginale congestionnée, et un écoulement clair et visqueux sort de la commissure inférieure de la vulve ».

Dans l'extériorisation du rut, le : rôle du phosphore semble prépondérant. Le même auteur note en effet l'action favorable d'une supplémentation en phosphate bicalcique à la dose de $30 \mathrm{~g}$ par jour et par tête.

Le régime intervient aussi de façon non équivoque sur la précocité de la puberté et l'âge auquel s'effectue le $1^{\text {er }}$ vêlage. DENIS, à Dahra (1971), note que l'âge moyen au $1^{\text {er }}$ vêlage est de 1.365,6 $\pm 240,0$ jours, soit en moyenne 3 ans et 9 mois pour des sujets soumis aux conditions naturelles mais abreuvés régulièrement, $1.077 \pm 98$ jours, soit 3 ans moins 18 jours pour un lot entretenu dans les parcs du Centre de Recherche zootechnique et $900 \pm 26$ jours, soit 2 ans et 5 mois sur le lot d'extériorisation des potentialités génétiques, abreuvé et nourri à volonté 
avec un régime supplémenté par un concentré titrant 0,90 UF et 120 à $130 \mathrm{~g}$ de M.A.D. au kg. Dans le même ordre d'idées, en Floride, PLASSE et Collab. (1968), observent des coïts fécondants dès l'âge de 19 mois.

\section{I.A.7.b) Température et état hygrométrique}

Le rôle de la température et de l'état hygrométrique est clairement démontré dans les observations faites à la station d'Entelbe en Uganda par MAHADEVAN et MARPLES (1961). Dans cette station équatoriale située à 1.100 mètres d'altitude, la saison sèche ne dure que trois mois (juin à août). Elle est suivie et précédée de deux périodes de forte pluviosité.

Contrairement à ce qu'on observe habituellement en zone tropicale, la période de plus grande fécondité se situe en saison sèche et au début de la saison des pluies qui la suit (de juillet à octobre). Elle correspond à la période pendant laquelle la température et l'état hygrométrique sont les plus favorables.

\section{II.A.7.c) Eclairement}

Le rôle des variations de la durée de l'éclairement est classiquement connu. Elles agissent sur le tractus génital par l'intermédiaire du relais hypothalamo-hypophysaire.

Chez le zébu, bien que discret en raison des faibles variations d'amplitude de la durée du jour en zone équatoriale, le rôle de l'éclairement semble devoir être évoqué à propos de l'observation de PAGOT au Niger, celle de PRIGENT et Collab. en Mauritanie et de celle de CUQ, FERNEY et VAN CRAEYNEST au Sénégal, qui notent tous un accroissement du taux des fécondations vers la fin du premier trimestre de l'année.

\section{II.A.7.d) Conclusion}

La vie génitale du zébu soumis aux conditions de l'élevage traditionnel est donc caractérisée par la fugacité, et la discrétion de l'œstrus, les âges relativement tardifs auxquels s'effectuent la puberté et le $1^{\text {er }}$ vêlage, le long écart qui sépare deux mise-bas successives et l'existence de saisons favorables à la reproduction.

L'importance que présentent les facteurs d'environnement, alimentation, température et hygrométrie en particulier, est telle qu'il nous a paru nécessaire d'approfondir nos connaissances sur les caractères de la vie génitale du zébu. Les résultats que nous avons obtenus font l'objet du chapitre d'histo-physiologie.

\section{II.B.) Etude histo-physiologique}

L'étude histo-physiologique que nous poursuivons depuis près de 4 ans a porté successivement sur les femelles (vides, ou gestantes) et les mâles.

\section{I.B.1.) LE CYCLE GSTRAL DE LA FEMELLE NON GESTANTE}

\section{II.B.1.a) Le prostrus}

Le prœstrus est caractérisé par les processus de maturation qui amènent un follicule du stock cavitaire petit et moyen au stade follicule mûr.

Généralement, un seul follicule cavitaire moyen subit l'évolution. Dans toutes nos observations, lorsque plusieurs la commencent, un seul la termine. Les autres involuent plus ou moins précocement. Cette particularité concorde avec la rareté des gestations gémellaires chez le zébu.

Parallèlement, pendant la période de l'œstrus, un certain nombre d'organites du stock des follicules primordiaux et primaires se transforment en follicules cavitaires petits et moyens. 


\section{II.B.1.b) L'estrus}

L'œestrus, chez Bos indicus, ne diffère pas, histologiquement, de l'œstrus de Bos taurus. Les images de déhiscence sont les mêmes. De la même façon, la cavité folliculaire est comblée par un caillot sanguin, avant qu'intervienne toute prolifération cellulaire de la thèque interne et de la granulosa.

\section{II.B.1.c) Le post estrus}

Le post cestrus correspond classiquement au développement et à la période d'activité du corps jaune (Corpus luteum).

Histologiquement le Corpus luteum subit pendant sa période d'activité des modifications successives que nous classons en trois types, pour les commodités de l'exposé. Le corps jaune en formation est caractérisé par l'envahissement rapide de la cavité folliculaire par des cordons cellulaires issus de la granulosa et de la thèque interne. Dans le corps jaune en activité, la totalité de la cavité folliculaire est comblée par les cordons cellulaires dont la masse est parcourue par de fines travées conjonctivo-vasculaires. Pendant la période de régression du corps jaune, les travées conjonctives s'épaississent et se multiplient. Elles se transforment en cloisons qui morcellent le parenchyme en îlots de plus en plus réduits.

Alors que chez Bos taurus, le corps jaune involue rapidement et se trouve toujours en état de régression avancée au début du prœstrus suivant, chez Bos indicus, l'involution s'effectue beaucoup plus lentement et de façon variable. Au début du cycle suivant nous avons, en effet, noté plusieurs fois la présence d'un corps jaune encore en état de pleine activité fonctionnelle.

\section{II.B.1.d) Le diøstrus}

Le diœstrus, du moins dans sa première moitié, est difficile à distinguer histologiquement du post-cestrus, en raison de la lenteur d'involution du corps jaune.

Le corps blanc est toujours d'apparition tardive. La dégénérescence hyaline, rapide et massive qui le caractérise, se produit au cours du cycle suivant. Pendant son involution, le corps blanc diminue peu à peu de volume. Il migre vers la zone parenchymateuse et finit par se fragmenter et disparaître. Des traces de corps blanc peuvent persister longtemps. Nous en avons observées dont l'origine pouvait être rapportée au $4^{\text {e }}$ cycle antérieur.

\section{II.B.1.e) Ancestrus}

L'observation la plus intéressante que nous ayons faite concerne la mise en évidence d'arrêts complets du cycle ou anastrus au sens strict du terme.

Ceux-ci sont caractérisés par l'absence d'organites pouvant être interprétés comme appartenant à un cycle en cours (follicules mûrs ou déhiscents et corps jaune). L'aspect morphologique des corps blancs qui proviennent des cycles antérieurs permet d'estimer la durée de l'ancestrus subi par les sujets.

Sur ces bases nous avons observé, outre des anœstrus courts (dont la durée est celle d'un cycle) et des ancestrus longs (pour lesquels toute trace de corps blancs antérieurs a disparu), toute une série de périodes de repos sexuels de durées intermédiaires.

Ces anœstrus intéressent 62 p. 100 des femelles vides observées de novembre 1970 à juillet 1971. Leur fréquence n'est donc pas négligeable.

La reprise de l'activité sexuelle après ces périodes de repos est fréquemment $(50 \mathrm{p} .100$ des cas) caractérisée par l'évolution maturative de plusieurs follicules cavitaires petits ou moyens ( 3 ou 4 le plus souvent) comme si, après une période réfractaire, le récepteur ovarien soumis à l'action d'une dose importante d'hormones gonadotropes ante-hypophysaires reprenait une activité intense dépassant les limites physiologiques du fonctionnement habituel.

\section{II.B.2.) FONCTIONNEMENT OVARIEN DES FEMELLES GESTANTES}

Chez Bos taurus, le corps gestatif maintient son activité pendant toute la gestation. Chez Bos indicus, au contraire, le corps gestatif de fécondation est souvent remplacé par d'autres. 
AU COURS DES TROIS PREMIERS MOIS DE LA GESTATION, le corps gestatif primaire s'organise et fonctionne.

PENDANT LES 2 MOIS QUI SUIVENT (4 et $5^{\mathrm{e}}$ mois de la gestation), sur 58 p. 100 des sujets nous avons noté l'existence d'un corps gestatif secondaire. Lorsque ce dernier apparaît, le corps gestatif primaire régresse et se transforme en corps blanc.

DU SIXIEME MOIS A LA FIN DE LA GESTATION, 41 p. 100 des femelles examinées présentent un corps gestatif tertiaire alors que 29 p. 100 conservent un corps gestatif secondaire actif et que 30 p. 100 n'ont pas remplacé leur corps gestatif primaire.

Sur quatre sujets, nous avons en outre noté, pendant cette période, des images nettes de maturation folliculaire se traduisant par la présence de gros follicules cavitaires, prélude possible à l'apparition d'une quatrième génération de corps gestatifs.

Le fonctionnement ovarien de la femelle zébu est donc caractérisé par plusieurs générations de corps gestatifs, par la lenteur de l'évolution des corps jaunes et surtout par l'existence de périodes d'ancstrus.

Cette dernière particularité explique l'irrégularité du cycle et l'existence de périodes favorables à la reproduction. Elle permet de classer au moins une partie des " cstrus à chaleurs silencieuses » dans la catégorie des ancestrus.

\section{II.B.3.) LE FONCTIONNEMENT GENITAL DU MALE}

Depuis deux ans, le travail que nous avons entrepris sur la femelle est complété par une étude de la vie génitale du mâle. Pour chaque sujet, nous avons effectué les prélèvements suivants : testicules, épididyme, canal déférent, vésicules séminales, prostate et glandes bulbouréthrales.

Les résultats que nous avons obtenus sur plus de 200 sujets sont identiques à ceux qui ont été publiés chez le bélier dont le cycle sexuel est saisonnier.

AU NIVEAU DU TESTICULE, aucune image suggérant une modification de la spermatogenèse n'a été enregistrée. La glande interstitielle, par contre, subit des variations quantitatives très nettes. Abondante pendant les périodes de repos sexuel, elle diminue en volume pendant les phases d'activité.

L'EPIDIDYME ET LE CANAL DEFERENT ne présentent aucune modification notable, sauf au niveau du renflement déférentiel dont les cellules prennent un caractère sécrétoire pendant les périodes d'activité.

Les VESICULES SEMINALES présentent, par contre, des différences très nettes. Leur épithélium est bas, non sécrétoire pendant les périodes de repos. Il est au contraire composé de cellules prismatiques hautes dont l'apex est bourré d'un matériel P.A.S. et bleu Alcian positif pendant les périodes d'activité.

Les Cellules des EPITHelia GLANDULAIRES DE LA PROSTATE ET DES GLANDES BULBO-URETHRALES présentent des modifications comparables à celles des vésicules séminales. $\mathrm{Si}$, morphologiquement, les différences sont délicates à apprécier, la mise en évidence histochimique des produits de sécrétion donne des résultats très nets.

Chez le taureau zébu comme chez le bélier, le rythme de reproduction n'affecte donc que « la suite fonctionnelle glandulaire ». La spermatogenèse s'effectue normalement mais les sécrétions nourricières et maturatrices font défaut pendant les périodes de repos sexuel.

\section{CONCLUSION}

Malgré d'importantes analogies, les appareils reproducteurs mâle et femelle du zébu présentent de nombreuses caractéristiques morphologiques et fonctionnelles. 
Celles-ci et en particulier l'existence de phases de repos dans les deux sexes condamnent toute assimilation trop hâtive avec Bos taurus. Le zébu n'est pas, comme beaucoup ont tendance à le croire, un bovin des régions tempérées adapté aux conditions des zones tropicales. Nombreux sont les caractères anatomiques et physiologiques qui lui sont propres. Il convient d'en tenir compte dans les travaux consacrés à la recherche des moyens capables d'augmènter sa productivité.

\section{SUMMARY}

\section{The anatomical functional basis of reproduction in zebu cattle}

The report contributed by the author has been founded upon some observations published by the zootechnicians who are in charge of the reproduction of the zebu cattle in the areas of extension of the species, and from the work done in the anatomical, histological and embryological Laboratory of the Ecole Inter-Etats des Sciences et Médecine Vétérinaires of the Université de Dakar.

In a chapter devoted to anatomy, the peculiarities of the male and female genital tract are treated.

The functional aspect of this problem is the subject of the second chapter in which the author first reports the zootechnical data referring to the duration of the cestrus, to the ages when puberty, the first fecund covering and the first calving occur, to the duration of gestation, to the interval which separates two consecutive calvings, to the annual rhythm of impregnations, and to the seasonal factors capable of having an influence on reproduction.

The histo-physiological study has permitted to emphasize the period of ancestrus in unimpregnated females, as well as three generations running of corpus luteum verum in pregnant females. For the male, the author points out some periods characterized by some aspects of the resting of appendant glands of the genital tract.

\section{RESUMEN}

\section{Bases anatómicas y funcionales de la reproducción en el cebú (Bos indicus)}

Este informe contiene las observaciones publicadas por los zootécnicos especializados en la reproducción del cebú en las áreas donde se encuentra esta especie y los trabajos efectuados en el laboratoire d'Anatomie, Histologie et Embryologie de l'Ecole Inter-Etats des Sciénces et Médecine Vétérinaires de l'Université de Dakar.

En una primera parte concerniendo a la anatomia, se notan las particularidades del tracto genital del macho y de la hembra.

El aspecto funcional del problema es el objeto de la segunda parte que comprende las datas zootécnicas tratando de la duración del ciclo estral, del estro, de las edades durante las cuales ocurren la pubertad, el primer apareamiento fecundo y el primer parto, de la duración de la gestación, del intervalo que separa dos partos consecutivos, del ritmo anual de las fecundaciones y de los factores estacionales capaz de influir en la reproducción.

El estudio histo-fisiológico permitió de poner en evidencia fases de anestro en las hembras no preñadas y tres generaciones sucesivas de cuerpos gestativos en las hembras preñadas.

En los machos, el autor señala periodos caracterizados por imagenes de reposo de glandulas anexas del tracto genital. 